TITLE:

\title{
Physical and chemical sputterings of solid surfaces irradiated by ethanol cluster ion beams
}

\author{
$\operatorname{AUTHOR}(\mathrm{S}):$ \\ Takaoka, Gikan H.; Kawashita, Masakazu; Okada, \\ Takeshi
}

\section{CITATION:}

Takaoka, Gikan H. ... [et al]. Physical and chemical sputterings of solid surfaces irradiated by ethanol cluster ion beams. REVIEW OF SCIENTIFIC INSTRUMENTS 2008, 79(2(Part 2)): 02 C503.

ISSUE DATE:

2008-02

URL:

http://hdl.handle.net/2433/84587

\section{RIGHT:}

Copyright 2008 American Institute of Physics. This article may be downloaded for personal use only. Any other use requires prior permission of the author and the American Institute of Physics. 


\title{
Physical and chemical sputterings of solid surfaces irradiated by ethanol cluster ion beams ${ }^{\text {a) }}$
}

\author{
Gikan H. Takaoka, ${ }^{\text {a) }}$ Masakazu Kawashita, and Takeshi Okada \\ Photonics and Electronics Science and Engineering Center, Kyoto University, Nishikyo, \\ Kyoto 615-8510, Japan
}

(Presented 28 August 2007; received 16 August 2007; accepted 2 November 2007; published online 5 February 2008)

\begin{abstract}
In order to clarify the interactions of ethanol cluster ion beams with solid surfaces, sputtering processes for $\mathrm{Si}, \mathrm{SiO}_{2}$ and $\mathrm{Au}$ surfaces were investigated by changing the acceleration voltage, the retarding voltage, and the incident angle. For the case of the normal incidence, the sputtered depth increased with increase of the acceleration voltage, and the sputtering ratio of $\mathrm{Si}$ to $\mathrm{SiO}_{2}$ was approximately 10 at an acceleration voltage of $9 \mathrm{kV}$. Furthermore, with regard to the incident angle dependence, the sputtered depth for the $\mathrm{Si}$ surfaces had a maximum value at an incident angle between $10^{\circ}$ and $60^{\circ}$. On the other hand, for the case of the physical sputtering by ethanol cluster ion irradiation on Au surfaces, the sputtered depth decreased with increase of the incident angle. With regard to the retarding voltage dependence, the sputtered depth had a maximum value for the $\mathrm{Si}$ surfaces, and the retarding voltage corresponding to the peak value changed according to the acceleration voltage applied. In addition, for the case of Au surfaces, the sputtered depth decreased with increase of the retarding voltage, and the physical sputtering was influenced by the minimum size of the cluster. (c) 2008 American Institute of Physics. [DOI: 10.1063/1.2819326]
\end{abstract}

\section{INTRODUCTION}

Collisions of cluster ions with solid surfaces have attracted much interest because they have exhibited specific features such as dissociation, chemical reaction, secondary electron emission, etc. ${ }^{1-5}$ With regard to the chemical reaction, the incident energy of the cluster ions is transferred in a surface collision, and chemical sputtering as well as physical sputtering is induced. On the other hand, liquid materials such as water and alcohol are well-known solvents. However, they cannot be used for etching the solid surfaces in conventional wet processes. While we have paid much attention to the unique properties of cluster ions, which are different from those of bulk state, we have produced liquid clusters such as water, alcohol, and paraffin clusters by an adiabatic expansion phenomenon. In addition, we have investigated the interactions of the liquid cluster ions on the solid surfaces. ${ }^{6-8}$

For the case of ethanol cluster ion irradiation, the impact of the cluster ions on the solid surfaces produced hydrogen and various kinds of alkyl radicals through the cluster ion fragmentation due to the high density irradiation effect. These radicals could drive the enhancement of surface reaction in chemical sputtering. ${ }^{8}$ In addition to the chemical sputtering, the physical sputtering also occurred through the multiple collisions, which were significantly different from the binary collision usually applied to monomer ion beam sputtering. As a result, high rate sputtering and smooth surface formation could be performed. ${ }^{9,10}$ For these sputtering pro-

\footnotetext{
a) Contributed paper, published as part of the Proceedings of the 12th International Conference on Ion Sources, Jeju, Korea, August 2007.

${ }^{a)}$ Electronic mail: gtakaoka@kuee.kyoto-u.ac.jp.
}

cesses, the incident energy and the cluster size of the ethanol cluster ions have an important role, and the sputtering phenomenon is also considered to change according to the incident angle. In this article, the sputtering effects of ethanol cluster ions on solid surfaces are investigated by changing the retarding voltage and the incident angle. The physical and chemical sputtering processes are discussed based on the cluster size distribution as well as the incident energy of the ethanol cluster ions.

\section{EXPERIMENTAL PROCEDURE}

The details of the liquid cluster ion beam system have been described elsewhere. ${ }^{6}$ Briefly, liquid materials such as ethanol were introduced into the cluster source and heated up to $150{ }^{\circ} \mathrm{C}$ by a wire heater attached around the source. The vapors of ethanol were ejected through a nozzle into a vacuum region. When the vapor pressure was larger than $1 \mathrm{~atm}$, the ethanol clusters were produced by an adiabatic expansion phenomenon. In the ionizer, the neutral clusters were ionized by electron bombardment. The cluster ions extracted from the ionizer were size separated by a retarding potential method. The size distribution of the ethanol cluster ions was controlled by adjusting the retarding voltage, and the minimum size of the cluster ions increased with increase of the retarding voltage. The size-separated cluster ion beams were accelerated toward a substrate, which was set on a substrate holder. The acceleration voltage $\left(V_{a}\right)$ was adjusted between 0 and $10 \mathrm{kV}$, and the retarding voltage $\left(V_{r}\right)$ was adjusted between 28 and $196 \mathrm{~V}$. The substrates used were 


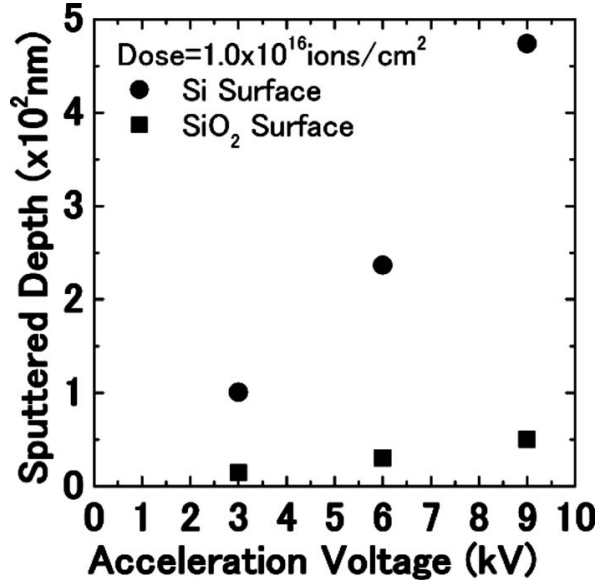

FIG. 1. Dependence of the sputtered depth for $\mathrm{Si}(100)$ and $\mathrm{SiO}_{2}$ surfaces on the acceleration voltage for the ethanol cluster ions at a normal incidence of $\theta=0^{\circ}$.

$\mathrm{Si}(100), \mathrm{SiO}_{2}$, and $\mathrm{Au}$ surfaces. The background pressure around the substrate was $1 \times 10^{-7}$ Torr, which was attained using a turbomolecular pump.

\section{RESULTS AND DISCUSSION}

The sputtering process by irradiation of ethanol cluster ions on substrate surfaces was investigated by changing the acceleration voltage and incident angle $(\theta)$ of the cluster ions. Figure 1 shows the dependence of sputtered depth for $\mathrm{Si}(100)$ and $\mathrm{SiO}_{2}$ surfaces on acceleration voltage for the ethanol cluster ions at a normal incidence of $\theta=0^{\circ}$. The retarding voltage was at $V_{r}=28 \mathrm{~V}$, and the cluster size used was larger than 100 molecules. The ion dose was 1.0 $\times 10^{16}$ ions $/ \mathrm{cm}^{2}$. As shown in the figure, the sputtered depth increases with increase of the acceleration voltage. When the acceleration voltage is $9 \mathrm{kV}$, the sputtered depth is $475 \mathrm{~nm}$ for $\mathrm{Si}$ and $47 \mathrm{~nm}$ for $\mathrm{SiO}_{2}$, respectively. The sputtering ratio of $\mathrm{Si}$ to $\mathrm{SiO}_{2}$ is approximately 10 at an acceleration voltage of $9 \mathrm{kV}$. The selectivity arises from the volatility of the reaction products and the difference in binding energy among the materials. This suggests that chemical reactions between $\mathrm{Si}$ and ethanol produce silicon hydride which is the dominant etching material for the Si surfaces.

Figure 2 shows the dependence of the sputtering yield for $\mathrm{Si}(100)$ and $\mathrm{Au}$ surfaces on the incident angle $(\theta)$ of the cluster ions. The retarding voltage was at $V_{r}=28 \mathrm{~V}$, and the cluster size used was larger than 100 molecules. The acceleration voltage was at $V_{a}=6$ and $9 \mathrm{kV}$. With regard to the chemical sputtering by ethanol cluster ion irradiation on $\mathrm{Si}$ surfaces, the sputtering yield has a maximum value at an incident angle between $10^{\circ}$ and $60^{\circ}$. The angle corresponding to the maximum peak increases with increase of the acceleration voltage. When the incident angle changes from normal to oblique, the chemical reaction frequency in the near surface region becomes high, and the ejected particles increase, resulting in high sputtering yield. However, for the ethanol cluster ion irradiation at higher incident angles, the incident energy of the cluster ions is partially deposited onto the substrate, and many molecules in the cluster are ejected or reflected from the substrate surface. Therefore, the sput-

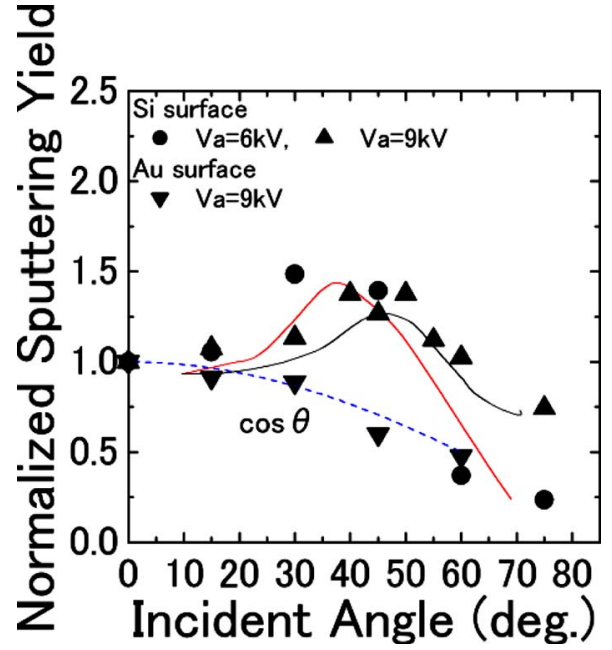

FIG. 2. (Color online) Dependence of the sputtering yield for $\mathrm{Si}(100)$ and $\mathrm{Au}$ surfaces on the incident angle $(\theta)$ of the cluster ions.

tering yield decreases with increase of the incident angle, and it has a peak at an incident angle between $10^{\circ}$ and $60^{\circ}$.

In contrast, for the case of the physical sputtering by ethanol cluster ion irradiation on Au surfaces, the sputtering yield decreases with increase of the incident angle, and it changes according to $\cos \theta$. For the case of the normal incidence, the penetration depth is the greatest, and most of the incident energy is deposited onto the substrate atoms. As a result, the sputtered atoms are ejected in the horizontal direction to the substrate surface due to the lateral sputtering effect. However, for the oblique incidence, the sputtered atoms are ejected in the forward direction of the cluster ion beams, and the atoms in the backward direction are redeposited on the substrate surface. Therefore, the sputtering yield decreases with increase of the incident angle for the $\mathrm{Au}$ surfaces.

Figure 3 shows the dependence of the sputtered depth for (a) $\mathrm{Si}(100)$ and (b) Au surfaces on the retarding voltage. Incident direction of the ethanol cluster ions was at a normal incidence. The ion dose was $1.0 \times 10^{15}$ ions $/ \mathrm{cm}^{2}$ for Si surface and $5.0 \times 10^{15}$ ions $/ \mathrm{cm}^{2}$ for Au surface, respectively. For the Si surfaces, the sputtering yield increases with increase of the retarding voltage. After it reaches a maximum value, it decreases with increase of the retarding voltage. On the other hand, for the Au surfaces irradiated by the ethanol

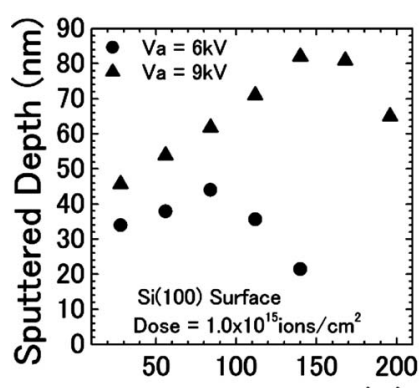

(a) Retarding Voltage (V)

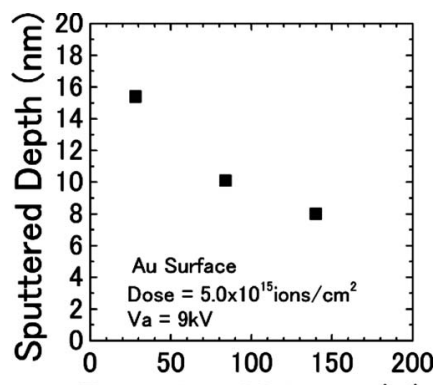

(b) Retarding Voltage (V)
FIG. 3. Dependence of the sputtered depth for (a) Si(100) and (b) Au surfaces on the retarding voltage for the ethanol cluster ions at a normal incidence of $\theta=0^{\circ}$. 
cluster ions, the sputtering yield decreases with increase of the retarding voltage. These results are related to the clustersize as well as the acceleration voltage, which suggests that the energy per molecule of the cluster ions influences the irradiation effects.

With regard to the size distribution of the ethanol cluster ions, the peak size was approximately 1000 molecules per cluster, and the minimum size was approximately 100 molecules per cluster at a retarding voltage of $28 \mathrm{~V}$.,9 In the previous study by using molecular dynamics simulation, the physical sputtering by the cluster ion beam irradiation was enhanced when the energy per molecule of the cluster ions was larger than $10 \mathrm{eV}$ per molecule. ${ }^{11}$ In contrast, the damage-free irradiation was achieved when the energy per molecule was less than a few $\mathrm{eV}$ per molecule. For the energy per molecule between a few $\mathrm{eV}$ per molecule and $10 \mathrm{eV}$ per molecule, the chemical sputtering is considered to have an important role in the irradiation effects. The amount of ethanol molecules, which are included in the energy range relating to the chemical sputtering irradiation, increases with increase of the retarding voltage, and it has a peak at a retarding voltage. This corresponds to the dependence of the sputtering yield for the Si surfaces on the retarding voltage. Based on these results, the chemical sputtering is predominant for the Si surfaces irradiated by the ethanol cluster ion beams.

${ }^{1}$ C. L. Cleveland and U. Landman, Science 257, 355 (1992).

${ }^{2}$ H. P. Cheng, C. L. Cleveland, and U. Landman, Science 260, 1304 (1993).

${ }^{3}$ W. Christen, U. Even, T. Raz, and R. D. Levine, Int. J. Mass Spectrom. Ion Process. 174, 35 (1998).

${ }^{4}$ S. Koizumi, H. Yasumatsu, S. Otani, and T. Kondow, J. Phys. Chem. A 106, 267 (2002).

${ }^{5}$ W. Christen and U. Even, Eur. Phys. J. D 24, 283 (2003).

${ }^{6}$ G. H. Takaoka, H. Noguchi, K. Nakayama, Y. Hironaka, and M. Kawashita, Nucl. Instrum. Methods Phys. Res. B 237, 402 (2005).

${ }^{7}$ G. H. Takaoka, H. Noguchi, K. Nakayama, T. Seki, and M. Kawashita, Rev. Sci. Instrum. 77, 03B508 (2006).

${ }^{8}$ G. H. Takaoka, K. Nakayama, T. Okada, and M. Kawashita, Proceedings of the 16th International Conference on Ion Implantation Technology (IIT06) (AIP, New York, 2006), p. 321.

${ }^{9}$ G. H. Takaoka, H. Noguchi, K. Nakayama, and M. Kawashita, e-J. Surf. Sci. Nanotechnol. 4, 473 (2006).

${ }^{10}$ G. H. Takaoka, M. Kawashita, K. Nakayama, and T. Okada, Nucl. Instrum. Methods Phys. Res. B 258, 209 (2007).

${ }^{11}$ T. Aoki, J. Matsuo, and G. H. Takaoka, Nucl. Instrum. Methods Phys. Res. B 202, 278 (2003). 